\title{
Detection Performance of Polarization and Spatial Diversities for Indoor GNSS Applications
}

\author{
Mohammadreza Zaheri, Ali Broumandan, Vahid Dehghanian, and Gérard Lachapelle
}

Schulich School of Engineering, Position Location and Navigation (PLAN) Group, University of Calgary, Calgary, AB, Canada T2N 1N4

Correspondence should be addressed to Ali Broumandan, abrouman@ucalgary.ca

Received 18 November 2011; Accepted 10 January 2012

Academic Editor: Hamsakutty Vettikalladi

Copyright ( 2012 Mohammadreza Zaheri et al. This is an open access article distributed under the Creative Commons Attribution License, which permits unrestricted use, distribution, and reproduction in any medium, provided the original work is properly cited.

\begin{abstract}
Multipath fading in the form of signal power fluctuation poses a formidable challenge to GNSS signal detection in harsh multipath environments such as indoors. Antenna diversity techniques such as polarization and spatial diversities can be used to combat multipath fading in wireless propagation channels. This paper studies and compares GPS signal detection performance enhancements arising from the spatial and polarization diversity techniques. Performance enhancements are quantified from a theoretical perspective and later verified based on several test measurements in various indoor environments. Enhancement is quantified based on measuring the correlation coefficient values between diversity branches, SNR levels, and computing the level crossing rate and average fade duration. In addition, the processing gain is quantified and the performance of each individual diversity system is evaluated. Experimental results show that, for a given target detection performance in terms of the probability of false alarm and the probability of detection, the required input SNR level to meet the target detection performance can be significantly reduced utilizing the diversity system.
\end{abstract}

\section{Introduction}

GNSS signal detection in indoor environments is a challenging problem since the signal suffers from insertion loss and fading. The magnitude of the insertion loss depends on the carrier frequency, the obstacle material, and the angle of incidence and can be as high as $30 \mathrm{~dB}[1,2]$. In addition to the insertion loss, the electromagnetic waves propagation in indoor environments also suffers from fading arising from the superposition of simultaneously arriving multipath signals with random phases and amplitudes. In reality, a moving handheld GNSS receiver experiences highly dynamic multipath situations resulting in high rate deep fading. In order to overcome these difficulties, various approaches such as HighSensitivity GPS (HSGPS) [3-6], Assisted GPS (AGPS) [7, 8], modernized GPS signals, and antenna diversity techniques $[9,10]$ have been developed.

Antenna diversity systems are establish based on the fact that multiple antennas with different patterns, polarizations, and locations provide multiple diversity branches such that the moment when the multipath fading affects the output of a diversity branch, another branch may have a reasonable signal power. Therefore, combining the independent copies of transmitted signals received by the diversity branches leads to less fading and higher overall SNR which improves the detection procedure. In antenna diversity systems, the receiver uses multiple antennas with different characteristics to collect statistically independent signals to establish a diversity system. These antennas can be different in terms of polarization or location, resulting in diversity systems known as polarization and spatial diversities, respectively. The signal detection enhancement utilizing a pair of spatially separated antennas is studied in [11]. It has been shown that a net processing gain can be achieved utilizing such a system. Colburn et al. [12] evaluate the spatial diversity performance for three different antenna configurations for the indoor $902-928 \mathrm{MHz}$ propagation channel. They show that the indoor propagation channel can be modeled by either a $\mathrm{Ri}$ cian or a Rayleigh distribution. The time and phase diversity techniques are studied in [13] and [14], respectively. 
Narayanan et al. [15] extensively analyse the polarization diversity and the channel characteristics at $1800 \mathrm{MHz}$ which is close to the L1 frequency. They analysed the effect of the correlation coefficient between two diversity branches on diversity gain thoroughly. In addition, they evaluated the performance of the polarization diversity using different antenna configurations in both Rayleigh and Rician fading environments. In [16], the performance of a dual channel spatial diversity system at $1800 \mathrm{MHz}$ has been considered. Lemieux et al. [17] have experimentally compared the space, frequency, and polarization diversities in the indoor environment for frequency of $900 \mathrm{MHz}$. Recently, au [18] proposed a new technique based on the motion of a single antenna to form a spatially distributed synthetic array for enhancing the detection performance of the GPS signals in the indoor environments.

The performance of dual-antenna spatial and polarization diversities to mitigate the multipath fading in the indoor environments is studied here. In the polarization diversity system, two structures have been considered. The first configuration consists of two circular polarized antennas, whereas the second one consists of two linear polarized antennas. A comparison is made based on the detection performances of each diversity scheme. Consequently, a detection scheme based on a Rayleigh fading channel is demonstrated and the processing gain realizable through utilizing spatial and polarization diversity techniques is quantified theoretically and experimentally. The second-order statistics of the multipath channel, namely, the Level Crossing Rate (LCR) and Average Fade Duration (AFD) metrics, are experimentally quantified and analyzed.

The remainder of this paper is organized as follows. The GPS signal structure and system model is described in Section 2. GPS signal polarization variations in multipath fading environments are also considered in this section. In Section 3, the signal detection procedure and the diversity approach utilized in this work is discussed and analyzed theoretically. The experiments performed are described in Section 4 and the empirical results are examined as well. Finally, Section 5 concludes the paper.

\section{Signal and Channel Model}

2.1. Signal Model. Herein, the demodulated complex baseband antenna output is denoted by $r(t)$. The signal component of $r(t)$ emanating from the $k$ th satellite is denoted as $s_{k}(t)$. This signal can be expressed as $s_{k}(t)=A_{k} s_{o_{k}}(t)$ where $s_{o_{k}}(t)$ is the deterministic complex baseband component of the $k$ th satellite signal that is known to the receiver and $A_{k}$ is the channel response to the incident signal. The received signal is corrupted with additive noise which has an equivalent complex baseband representation denoted by $w(t)$. It is assumed that $w(t)$ is a complex normal random process, independent of the signal with a power spectral density (PSD) that is constant within the bandwidth of the received signal with a level of $N_{o}$. Therefore, $r(t)$ can then be expressed as

$$
r(t)=S(t)+w(t)
$$

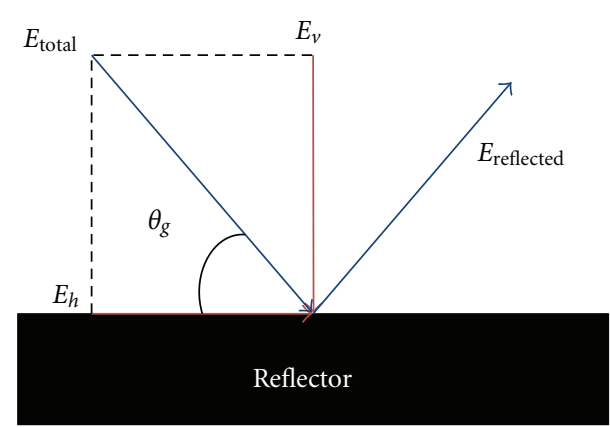

FIGURE 1: Reflected electromagnetic signal.

where

$$
S(t)=\sum_{k=1}^{K} A_{k}(\mathbf{p}(t)) D_{k}\left(t-\tau_{k}\right) c_{k}\left(t-\tau_{k}\right) e^{j\left(2 \pi \Delta f_{k} t+\psi_{k}\right)} .
$$

$A_{k}(\mathbf{p}(t))$ is the complex channel gain which is a function of the antenna position $\mathbf{p}(t), D_{k}(t)$ is the navigation data modulation, $c_{k}(t)$ is the PRN code, $\tau_{k}$ is the code phase, $\Delta f_{k}$ represents the carrier frequency offset, $\psi_{k}$ is the initial phase offset, $K$ is the number of satellites in view, and $w(t)$ is complex additive white Gaussian noise. Herein, the signal detection of individual GNSS satellites will be considered, while the presence of simultaneous GNSS signals is not considered. This is due to the fact that the cumulative selfinterference from other PRNs is negligible compared to other dominant independent noise sources [19]. Hence, in the remainder of the text, for the notational convenience, the subscript of $k$ is ignored and the received signal is modeled as

$$
r(t)=A(\mathbf{p}(t)) s_{o}(t)+w(t),
$$

where $s_{o}(t)=D(t-\tau) c(t-\tau) e^{j(2 \pi \Delta f t+\psi)}$ is known to the receiver except for the navigation data $D(t)$, the code phase $\tau$, the carrier frequency offset $\Delta f$, and the initial phase offset $\psi$. In unresolvable Rayleigh-faded multipath environments which is assumed here, $A(\mathbf{p})$ can be modeled by a Complex Normal $(\mathrm{CN})$ random variable, such that $A \sim \mathrm{CN}\left(0, \sigma_{A}^{2}\right)$ where $\sim$ denotes the PDF of the left-hand side variable and $\mathrm{CN}\left(\mu, \sigma^{2}\right)$ signifies a complex normal probability density function (PDF) of mean $\mu$ and variance $\sigma^{2}$ [20].

The received signal passes through the signal acquisition procedure as an initial operation dealing with the detection and coarse estimation of Doppler and code phase offset. A two-dimensional search is applied on the signal to roughly estimate the Doppler frequency and the code phase offset.

\subsection{GPS Signal Polarization in Dense Multipath Environ-} ments. The transmitted GPS signal is a right hand circularly polarized (RHCP) electromagnetic (EM) wave which is a special case of the more general elliptically polarized wave. Assume that an RHCP GPS signal reaches the reflector with an incident angle (grazing angle) of $\theta_{g}$ as shown in Figure 1. For a circularly polarized signal, the electric field intensity vector $\mathbf{E}$ is decomposed into two orthogonal components, 
$E_{h}$ and $E_{v}$, called horizontal and vertical components, respectively. Since the horizontal and vertical components of the reflection coefficient of a reflector are not equal [20], the absolute value of the $\mathbf{E}$ field components in the reflected signal changes independently. Therefore, the circular polarity of the reflected GPS signal changes to elliptical. In addition to magnitude, the phase of each component changes depending on the Brewster angle, $\Psi_{B}$ (known as the polarization angle as well) as a property of the reflectors [21]. For instance, Brewster angle for a metallic reflector is a few degrees [21] while for a concrete surface is around 69 degrees [22]. After reflection, the phase of the horizontal component $\left(E_{h}\right)$ always changes by 180 degrees; however, that of the vertical component $\left(E_{v}\right)$ may change either by 0 or 180 degrees depending on whether the grazing angle is below or above the Brewster angle [21]. If the grazing angle is higher than the Brewster angle, the vertical component phase changes by 180 degrees and the reflected signal has right hand polarization. On the other hand, if the grazing angle is lower than the Brewster angle, the reflected signal has left-hand polarization. Hence, the reflected wave may have either left- or right-hand elliptical polarization based on the impinging angle. Balanis [23] shows that an elliptical polarized signal can be written as a combination of two RHCP and LHCP waves. Hence, if the grazing angle of the signal is less than the Brewster angle, the RHCP component of the reflected signal is stronger whereas, if the grazing angle of the LOS signal is greater than the Brewster angle, the LHCP component of the reflected signal will be stronger. In indoor environments, since the received signals are highly likely to be subject to reflections, the final received signals can be assumed to be elliptically polarized and, hence, include both RHCP and LHCP components. The arriving RHCP and LHCP waves arise from the superposition of many reflected waves. Since the reflection phenomenon is random, the final RHCP and LHCP waves are uncorrelated and, therefore, can be used for diversity gain. This diversity gain is quantified through theoretical analysis and verified based on actual measurements in this paper.

\section{Diversity Scheme and Detection Procedure}

Having collected GPS signals with the diversity branches, the received signals should be combined using an efficient combiner. Herein, the main focus is to combine signals received from two diversity branches. In order to implement a detector, the likelihood ratio test (LRT) function is utilized. The detection problem is to find a moment in each code and Doppler search where the incoming signal and the locally generated one are synchronized. Two hypotheses $H_{0}$ and $H_{1}$ are defined as

$$
\begin{aligned}
& H_{0}: x[m]=\omega[m], \quad m=1,2, \\
& H_{1}: x[m]=s[m]+\omega[m], \quad m=1,2 .
\end{aligned}
$$

Here, $m$ is the number of diversity branches, $x[m]$ is the correlator output, $s[\mathrm{~m}]$ is the desired signal in which the code phase and Doppler of the incoming signal and the locally generated one are synchronized, $\omega[\mathrm{m}]$ is the embedded zero mean white complex Gaussian noise with a covariance matrix of $\mathbf{C}_{\mathbf{w}}=\sigma_{\omega}^{2} \mathbf{I}_{2}$ where $\sigma_{\omega}^{2}$ is the noise variance, and $\mathbf{I}_{2}$ is $2 \times 2$ identity matrix. In a Rayleigh-faded channel, the desired signal received at the antenna is a zero mean circular normal distribution with a covariance matrix of $\mathbf{C}_{\mathbf{s}}$. Therefore, the signal probability distribution function under $H_{0}$ and $H_{1}$ hypotheses can be defined as

$$
\begin{aligned}
& p\left(\mathbf{x} ; H_{0}\right) \sim \mathrm{CN}\left(0, \mathbf{C}_{\mathbf{w}}\right), \\
& p\left(\mathbf{x} ; H_{1}\right) \sim \mathrm{CN}\left(0, \mathbf{C}_{\mathbf{s}}+\mathbf{C}_{\mathbf{w}}\right) .
\end{aligned}
$$

Under these conditions, the final test statistic becomes [24]

$$
T(\mathbf{x})=\mathbf{x}^{H} \mathbf{C}_{s}\left(\mathbf{C}_{s}+\sigma_{w}^{2} \mathbf{I}_{2}\right)^{-1} \mathbf{x} .
$$

In a general case, the received signal from different diversity branches may be correlated. In order to simplify the final test statistic represented in (6), the modal matrix $\mathbf{V}$, a $2 \times$ 2 matrix formed with the eigenvectors of $\mathbf{C}_{s}$, is used to decorrelate the input signals by $\mathbf{y}=\mathbf{V}^{T} \mathbf{x}$. The $\mathbf{V}$ matrix is composed of eigenvectors of $\mathrm{C}_{s}, \mathrm{~V}^{\mathrm{H}} \mathrm{C}_{\mathrm{s}} \mathbf{V}=\boldsymbol{\Lambda}_{\mathrm{s}}$, where $\boldsymbol{\Lambda}_{\mathrm{s}}$ is a matrix of eigenvalues of $\mathbf{C}_{s}$ and is formed as

$$
\Lambda_{\mathbf{s}}=\left[\begin{array}{cc}
\lambda_{s 1} & 0 \\
0 & \lambda_{s 2}
\end{array}\right] .
$$

Therefore, the covariance matrix of $y$ becomes

$$
\begin{aligned}
\mathbf{C}_{\mathbf{y}} & =\mathbf{E}\left[\mathbf{y} \mathbf{y}^{\mathbf{H}}\right]=\mathbf{V}^{\mathrm{H}} \mathbf{C}_{\mathbf{x}} \mathbf{V} \\
& =\mathbf{V}^{\mathrm{H}}\left(\mathbf{C}_{\mathbf{s}}+\sigma_{w}^{2} \mathbf{I}_{2}\right) \mathbf{V} \\
& =\mathbf{V}^{\mathrm{H}} \mathbf{C}_{\mathbf{s}} \mathbf{V}+\sigma_{w}^{2} \mathbf{V}^{\mathrm{H}} \mathbf{V} \\
& =\boldsymbol{\Lambda}_{\mathbf{s}}+\sigma_{w}^{2} \mathbf{I}_{2} .
\end{aligned}
$$

After some manipulations, the final test statistic is reduced to [24]

$$
T\left(\mathbf{y}=\mathbf{V}^{\mathrm{T}} \mathbf{x}\right)=\sum_{m=1}^{2} \frac{\lambda_{s m}}{\lambda_{s m}+\sigma_{w}^{2}} y_{m}^{2}
$$

Consequently, the final test statistic can be considered as a decorrelator followed by a weighting function as shown in Figure 2. This detector is known as the Estimator-Correlator (EC).

3.1. Detection Performance Evaluation. The detector performance is evaluated by the probability of false alarm $P_{\mathrm{FA}}$ and the probability of detection $P_{D}$. The probabilities of false alarm and detection for the final test statistic in (9) are quantified as

$$
\begin{gathered}
P_{\mathrm{FA}}(\lambda)=\int_{y=\lambda}^{\infty} p\left(T(y) ; H_{0}\right) d y, \\
P_{D}(\lambda)=\int_{y=\lambda}^{\infty} p\left(T(y) ; H_{1}\right) d y,
\end{gathered}
$$

respectively, where $p\left(T(y) ; H_{i}\right)$ is the probability function of $T(y)$ under the $H_{i}$ condition and $\lambda$ is a threshold defining 


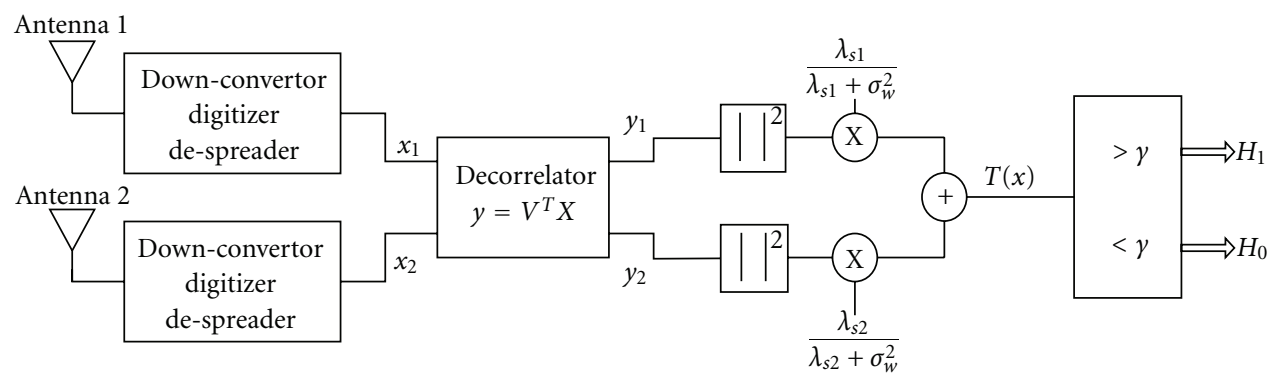

FIGURE 2: LRT detector scheme for a two-antenna diversity system.

the $P_{D}$ and $P_{\mathrm{FA}}$. The probability function of $\mathbf{y}=\mathbf{V}^{\mathrm{T}} \mathbf{x}$ can be written as

$$
\begin{aligned}
& p\left(\mathbf{y} ; H_{0}\right) \sim \mathrm{CN}\left(0, \mathbf{C}_{\mathbf{w}}\right), \\
& p\left(\mathbf{y} ; H_{1}\right) \sim \mathrm{CN}\left(0, \boldsymbol{\Lambda}_{\mathbf{s}}+\mathbf{C}_{\mathbf{w}}\right) .
\end{aligned}
$$

Thus, y has a zero mean Gaussian distributions and $|y|^{2}$ results in central chi-squared distributions. Hence, the characteristics function of $T(y)$ leads to the summation of two weighted central chi-squared distributions. The probabilities of detection and false alarm for this model are quantified as [25]

$$
\begin{aligned}
P_{D} & =\int_{\gamma}^{\infty} \int_{-\infty}^{\infty} \frac{1}{\left(1-j \lambda_{s 0} \omega\right)\left(1-j \lambda_{s 1} \omega\right)} \exp (-j \omega t) \frac{d \omega}{2 \pi} d t, \\
P_{\mathrm{FA}} & =\int_{\gamma}^{\infty} \int_{-\infty}^{\infty} \frac{1}{\left(1-2 j \alpha_{0} \omega\right)\left(1-2 j \alpha_{1} \omega\right)} \exp (-j \omega t) \frac{d \omega}{2 \pi} d t,
\end{aligned}
$$

where

$$
\alpha_{n}=\frac{\lambda_{s n} \sigma_{w}^{2}}{\lambda_{s n}+\sigma_{w}^{2}} .
$$

To determine a closed-form expression for $P_{\mathrm{FA}}$ and $P_{D}$ in the case of distinctive eigenvalues, it can be shown, using a partial fraction expansion, that (13) can be reduced to [24]

$$
P_{D}=\frac{\lambda_{s 2} e^{-\gamma / \lambda_{s 2}}-\lambda_{s 1} e^{-\gamma / \lambda_{s 1}}}{\lambda_{s 2}-\lambda_{s 1}} .
$$

Similarly, the $P_{\mathrm{FA}}$ can be computed as

$$
P_{\mathrm{FA}}=\frac{\alpha_{2} e^{-\gamma / \alpha_{2}}-\alpha_{1} e^{-\gamma / \alpha_{1}}}{\alpha_{2}-\alpha_{1}},
$$

where

$$
\alpha_{n}=\frac{\lambda_{s n} \sigma_{w}^{2}}{\lambda_{s n}+\sigma_{w}^{2}}, \quad n=1,2 .
$$

3.2. Theoretical Analysis of the Indoor Environment. In the dual-branch diversity system, a general scenario for the received signal covariance matrix can be defined as

$$
\mathbf{C}_{\mathbf{s}}=\left[\begin{array}{cc}
\sigma_{s_{1}}^{2} & E\left(s_{1} s_{2}^{*}\right) \\
E\left(s_{2} s_{1}^{*}\right) & \sigma_{s_{2}}^{2}
\end{array}\right]
$$

where $x_{1}$ and $x_{2}$ are the correlator outputs of the input branches, $\sigma_{s_{m}}^{2}=E\left(s_{m} s_{m}^{*}\right)$ is the variance of $s_{m}$ under $H_{1}$ condition for $m=\{1,2\},(\cdot)^{*}$ is a conjugate operator, and $E\left(s_{1} s_{2}^{*}\right)=E\left(s_{2} s_{1}^{*}\right)$. To simplify the general equation for the signal covariance matrix, without loss of generality, assume that

$$
\sigma_{s_{1}}^{2}=\sigma_{s}^{2}, \quad \sigma_{s_{2}}^{2}=r \sigma_{s}^{2},
$$

where $r=\sigma_{s 2}^{2} / \sigma_{s 1}^{2}$ is the power ratio of the input signals. In addition, the cross-correlation coefficient $(\rho)$ between the received signals is quantified as

$$
\rho=\frac{E\left(s_{1} s_{2}^{*}\right)}{\sqrt{\operatorname{var}\left(s_{1}\right) \operatorname{var}\left(s_{2}\right)}}=\frac{E\left(s_{1} s_{2}^{*}\right)}{\sqrt{\sigma_{s}^{2} \times r \sigma_{s}^{2}}}=\frac{E\left(s_{1} s_{2}^{*}\right)}{\sigma_{s}^{2} \sqrt{r}} .
$$

Therefore, the signal covariance matrix can be rewritten as

$$
\mathbf{C}_{\mathbf{s}}=\sigma_{s}^{2}\left[\begin{array}{cc}
1 & \rho \sqrt{r} \\
\rho \sqrt{r} & r
\end{array}\right] .
$$

As shown in (9), the detection test statistics is a function of the eigenvalues of the signal covariance matrix $\lambda_{s 1}$ and $\lambda_{s 2}$. In this case, the eigenvalues of the covariance matrix are [26]

$$
\begin{aligned}
& \lambda_{s 1}=\frac{\sigma_{s}^{2}}{2}\left[(r+1)+\sqrt{(1-r)^{2}+4 r \rho^{2}}\right], \\
& \lambda_{s 2}=\frac{\sigma_{s}^{2}}{2}\left[(r+1)-\sqrt{(1-r)^{2}+4 r \rho^{2}}\right] .
\end{aligned}
$$

The probability of detection for a fixed probability of false alarm $\left(P_{\mathrm{FA}}=0.01\right)$ as a function of the signal cross correlation coefficient and the input power difference is shown in Figure 3. Lower performance results from a higher power difference and cross-correlation coefficient between input branches. The interesting observation is that for the correlation coefficient values less than 0.4 the $P_{D}$ for given $P_{\mathrm{FA}}$ does not significantly degrade.

In the case of uncorrelated equal power input signals ( $\rho=$ 0 and $r=1$ ), the EC detector is simplified to equal gain (EG) combiner as

$$
T(\mathbf{x})=x_{1}^{2}+x_{2}^{2}
$$




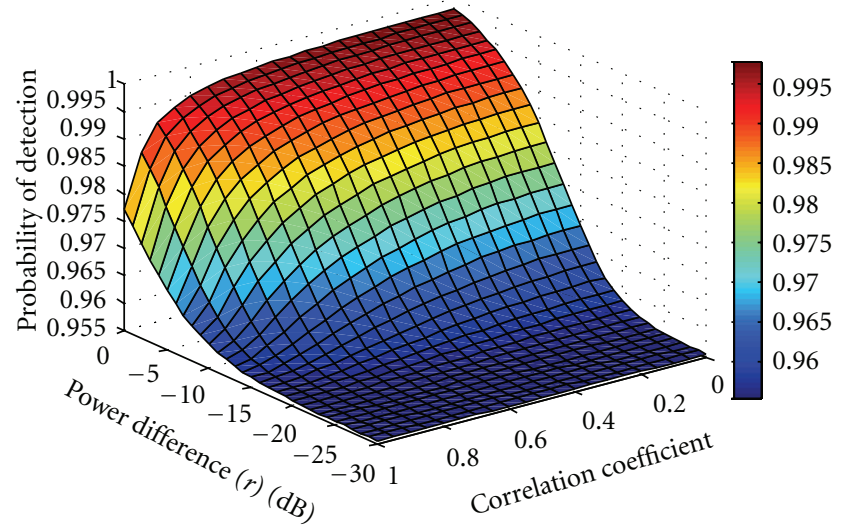

Figure 3: $P_{D}$ for $P_{\mathrm{FA}}=0.01$ for the EC combiner versus correlation coefficient and power difference between diversity branches.

However, combining the correlated unequal power signals by the equal gain (EG) combiner results in the probability of false alarm and probability of detection given by

$$
\begin{gathered}
P_{\mathrm{FA}}=\left(1+\frac{\gamma}{\sigma_{\omega}^{2}}\right) e^{-\gamma / \sigma_{\omega}^{2}}, \\
P_{D}=\frac{\lambda_{x 2} e^{-\gamma / \lambda_{x 2}}-\lambda_{x 0} e^{-\gamma / \lambda_{x 1}}}{\lambda_{x 2}-\lambda_{x 1}},
\end{gathered}
$$

respectively, where $\lambda_{x 1}$ and $\lambda_{x 2}$ are the eigenvalues of $\mathbf{C}_{\mathbf{x}}=$ $\mathbf{C}_{\mathbf{s}}+\sigma_{w}^{2} \mathbf{I}_{2}$ and can be shown to be

$$
\begin{aligned}
& \lambda_{x 1}=\frac{1}{2}\left[\sigma_{s}^{2}(r+1)+2 \sigma_{w}^{2}+\sigma_{s}^{2} \sqrt{(1-r)^{2}+4 r \rho^{2}}\right], \\
& \lambda_{x 2}=\frac{1}{2}\left[\sigma_{s}^{2}(r+1)+2 \sigma_{w}^{2}-\sigma_{s}^{2} \sqrt{(1-r)^{2}+4 r \rho^{2}}\right] .
\end{aligned}
$$

These will be used in the following section to compare the diversity gain performance of EC and EG combiners.

3.3. Diversity Gain Evaluation. The effectiveness of a diversity system is evaluated by a quantity known as the diversity gain. Herein, the diversity gain is defined as the excess in the required input average SNR for a single antenna scheme to achieve the same $P_{D}$ as the combining scheme for a specific $P_{\mathrm{FA}}$. For a given target detection performance in terms of $P_{D}$ and $P_{\mathrm{FA}}$, the diversity gain is defined as

$$
G=\frac{\mathrm{SNR}_{S}}{\mathrm{SNR}_{D}},
$$

where $\mathrm{SNR}_{S}$ and $\mathrm{SNR}_{D}$ signify the required $\mathrm{SNR}$ values for the single branch and the diversity combiner, respectively, to achieve the same detection performance.

Figure 4 shows the diversity gain as a function of the cross-correlation coefficient and power difference between the diversity branches for a specific design point of $P_{\mathrm{FA}}=$ 0.01 and $P_{D}=0.9$ using the estimator correlator (EC) as the detector. The results of Figure 4 show that up to $5.5 \mathrm{~dB}$ diversity gain for uncorrelated and equal power input signals can be achieved. As shown by increasing the correlation

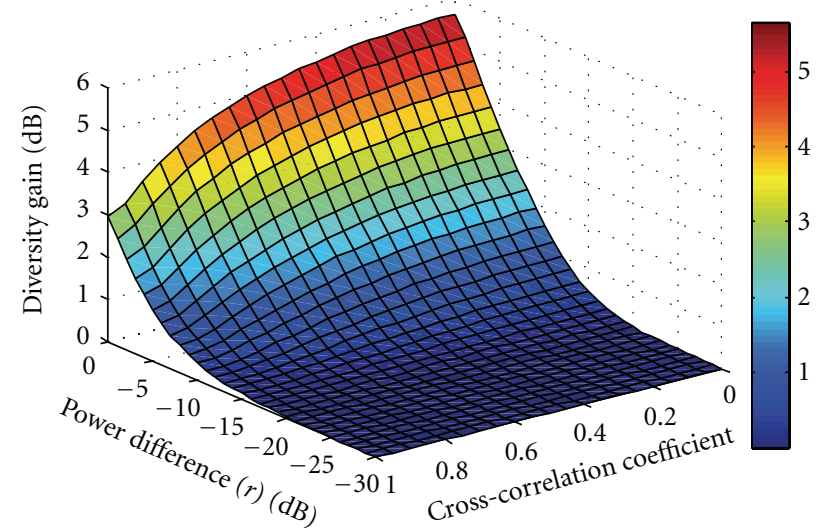

FIGURE 4: Diversity gain versus correlation coefficient and power difference between diversity branches.

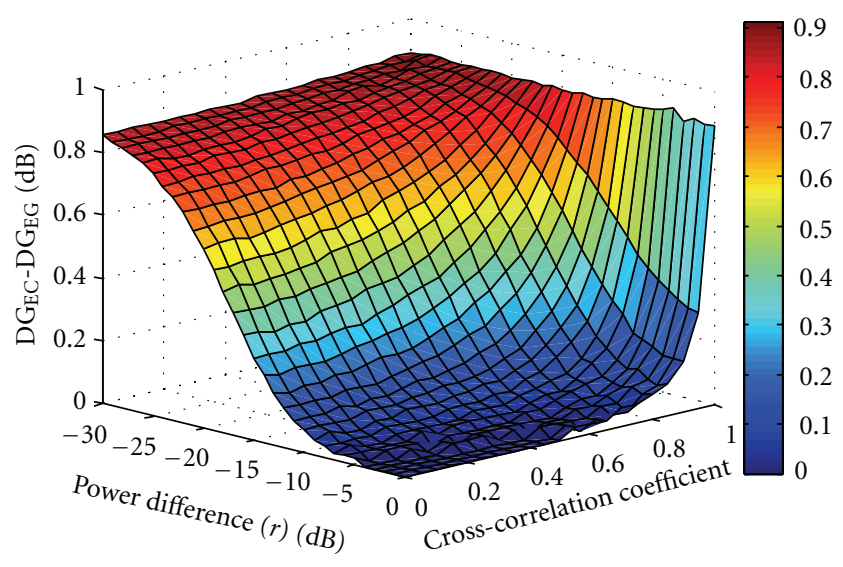

FIGURE 5: EC and EG diversity gain difference versus correlation coefficient and power difference between diversity branches.

coefficient between branches, the diversity gain declines. However, by increasing the power difference between input signals, the diversity gain reduces to $0 \mathrm{~dB}$ even for uncorrected input signals, which is due to the signal degradation in one of the branches.

In Figure 5, the diversity gain difference between the estimator correlator (EC) and equal gain (EG) combiner is shown as a function of power difference and crosscorrelation coefficient between the diversity branches. Here, the design point is defined as $P_{\mathrm{FA}}=0.01$ and $P_{D}=0.9$. Figure 5 shows that EC and EG result in the same detection performance for low correlation coefficient between the input branches (up to 0.4).

\section{Experimental Results}

In the previous sections, the detection performances of the diversity systems in multipath fading environments modeled by the Rayleigh fading were theoretically examined. The experimental measurements described in this section validate the assumptions in the context of the GNSS signal detection in indoor environments. The objective of the experimental measurements conducted herein is to examine 


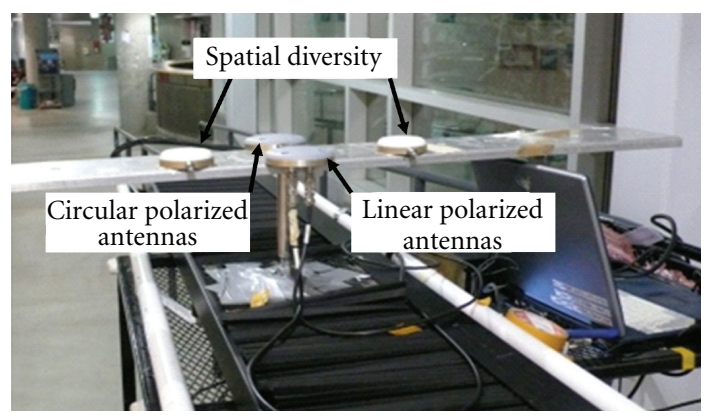

FIGURE 6: Three antenna diversity structures mounted on a moving linear table.

the dual-antenna diversity systems, namely, the polarization and spatial diversities for a selection of typical indoor locations.

4.1. Test Setup. A set of experiments was performed in order to compare the performance of the polarization and spatial diversity structures. Measurement results in [18, 27] have shown that dense indoor GNSS channels decorrelate by almost half a carrier wavelength. Hence, herein spatial diversity was achieved by two spatially RHCP antennas separated by 1.5 wavelength of the GPS L1 signal $(30 \mathrm{~cm})$. The spatial diversity main principle is based on the fact that in dense multipath environments the received signals are a random function of antenna location. Herein, a dual circular polarized antenna consisting of RHCP and LHCP components was used to implement circular polarization diversity. More details regarding the utilized antennas can be found in [28]. Besides, a dual-polarized antenna consisting of two linear polarized antennas mounted on a horizontal plane and mutually perpendicular, called vertical and horizontal antenna, was utilized to create a linear polarization diversity structure. The principle of this diversity structure is based on the fact that the amplitude and the phase of the vertical and horizontal components of the reflected signals vary independently and can be considered as a diversity structure.

In order to achieve the objectives of this work, two datasets in two different indoor locations were collected. Each dataset consisted of three consecutive data collections with different diversity schemes, namely, the circular polarization (CP), spatial polarization (SP), and linear polarization (LP) structures, as shown in Figure 6. In each case, the diversity branches along with a reference antenna were connected to a synchronized triple port downconverter/digitizer to collect synchronous raw IF samples in the GPS L1 band. The indoor antennas were mounted on a linear motion table to collect independent signal samples in the indoor GPS channels. The table moved by about $2.8 \mathrm{~m}$ in each lap with an antenna speed of $2 \mathrm{~cm} / \mathrm{s}$. The first data collection set was performed in a laboratory with a high metallic ceiling as shown in Figure 7(a). The second dataset was carried out in the corridor of a building having large window panes in both the south and north directions and a concrete ceiling as shown in Figure 7(b). To aid the indoor receiver process with the navigation data, a reference antenna was located in an open-sky condition within 30 metres of the indoor diversity structures. The reference channel was used to remove the navigation data bit from the received signals to increase the coherent integration time up to $100 \mathrm{~ms}$. The GSNRx-rr software GNSS receiver [29] was used to process the data.

4.2. Comparison of the Polarization and Spatial Diversities. In this section, different metrics have been used to characterize different antenna diversity systems.

4.2.1. Correlation Coefficient. As shown earlier, the correlation coefficient between diversity branches can be used to characterize the performance of a diversity system. Narayanan et al. in [15] quantify both complex and envelope correlation coefficient and conclude that the value of complex correlation coefficients is constant from run to run and hence it is a reliable metric. Herein, the complex correlation coefficient between the received signals in each diversity branch is quantified as [12]

$$
\rho_{c}=\left|\frac{E\left[x_{1} x_{2}^{*}\right]}{\sqrt{E\left[x_{1} x_{1}^{*}\right] E\left[x_{2} x_{2}^{*}\right]}}\right|,
$$

where $E[\cdot]$ is the expected value operator. The results of correlation coefficient measurements for circular polarization (CP), spatial and linear polarization (LP) diversity systems, and different PRNs are shown in Figure 8. According to the results, the correlation coefficient for the majority of the datasets is less than 0.3 , which results in a diversity gain. Considering the results of Figure 5, the estimator-correlator and equal-gain combiner performance does not have any significant difference for the correlation coefficient values less than 0.4 .

4.2.2. Average Input SNR. As mentioned before, the average input SNR plays a crucial role in the efficiency of a diversity system. The measured carrier-to-noise ratio $\left(\mathrm{C} / \mathrm{N}_{0}\right)$ values of the received signals through different diversity branches are quantified and shown in Figure 9. GSNRx-rr was used to measure the $\mathrm{C} / \mathrm{N}_{0}$ values. Considering the results of Figure 9, the level difference of the received signals in the different diversity branches is not considerable and, given the theoretical results (Figure 5), is negligible. According to these findings and considering the results of Figure 5, the equal gain combiner is considered as the combining method herein. The interesting observation is that in all cases the received signal power through the LHCP antenna is slightly more than that of the RHCP antenna. As explained before, this implies that the LHCP component of the reflected signals is stronger than the RHCP component. Thus, considering the LHCP component as an input for the circular polarization, diversity leads to better signal detection performance.

4.2.3. Average Fade Duration and Level Crossing Rate. In indoor GPS multipath channels modeled by Rayleigh fading, the received signal amplitude is subject to rapid fluctuations. As the GPS receiver moves, the rate of change of amplitude 


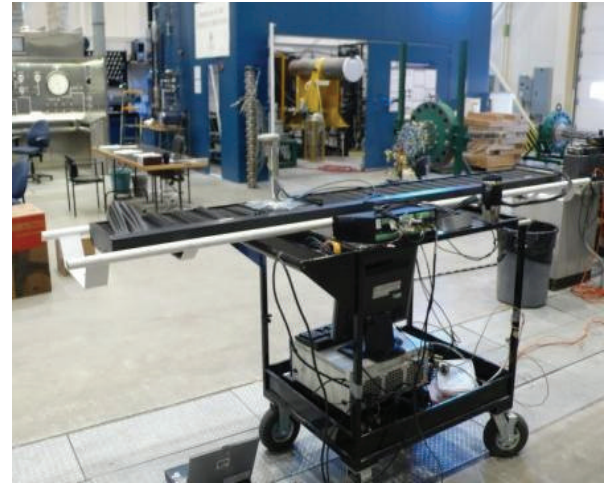

(a)

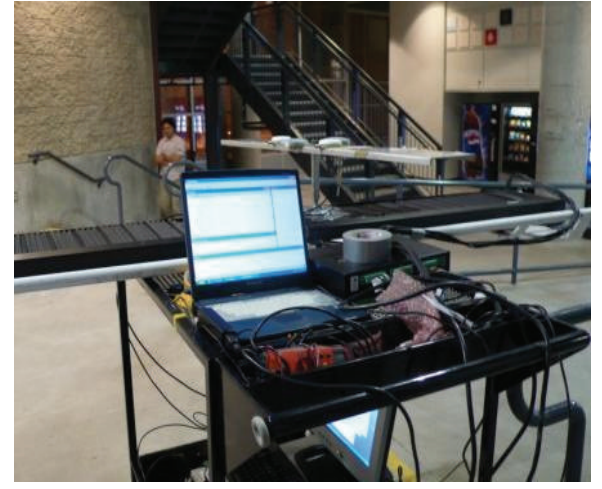

(b)

Figure 7: Data collection sites in indoor locations (a) Energy High Bay (b) corridor.

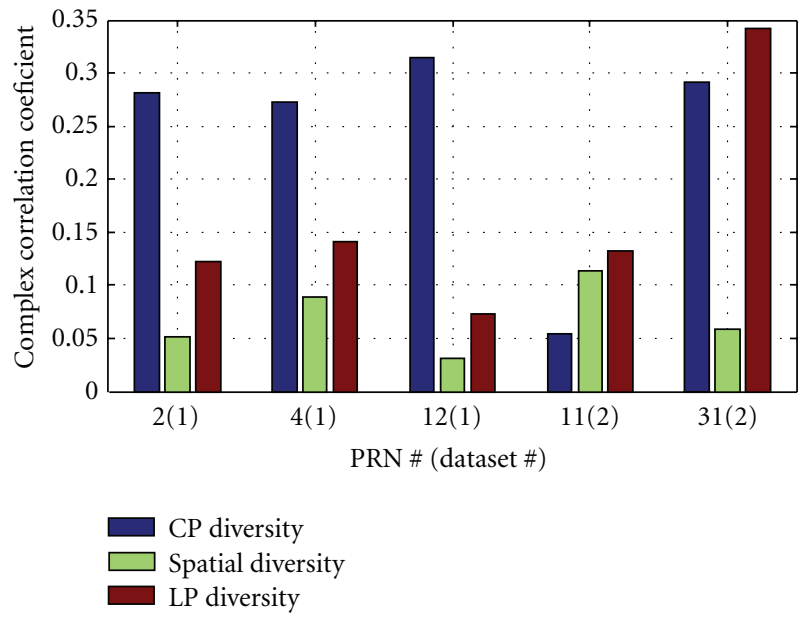

Figure 8: Correlation coefficients between two diversity branches for different diversity structures.

changes. The Rayleigh fading distribution just describes the first-order statistics of the signal amplitude for short distances where the mean level of the received signal can be assumed constant. The first-order statistics does not provide any information regarding the fading time or distance. It just provides an overall percentage of time or location where the signal amplitude lies below a specific threshold. Hence, it is of interest to characterize the rate at which fades of any depth happen and the average fade duration below any given depth. In indoor GPS multipath channels, the fading envelope varies and the fading rate and envelope amplitude are a function of time. In order to obtain a quantitative measure of the fading occurring in the channel, the level crossing rate (LCR) and the average fade duration (AFD) are quantified as two statistical parameters of the channel model [30]. LCR is quantified as the expected rate at which the received signal envelope crosses a specific level, in either the positive or negative direction. The AFD is the average fade duration below any given depth. In other words, AFD is quantified as the average period of time for which the received signal envelope is below a specific level.

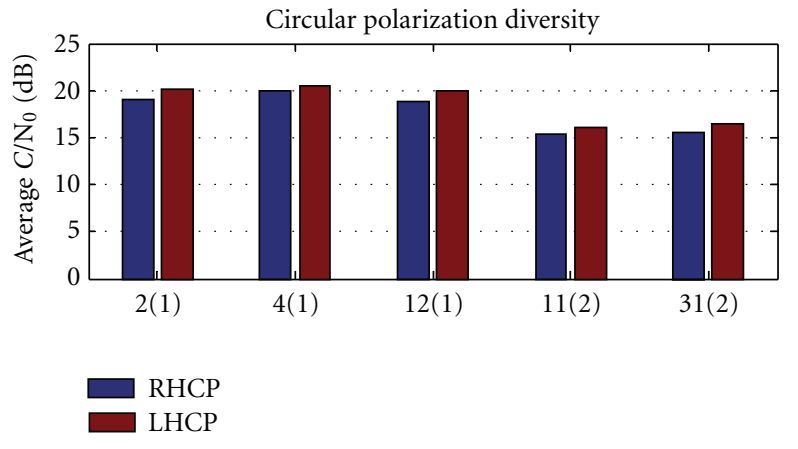

(a)

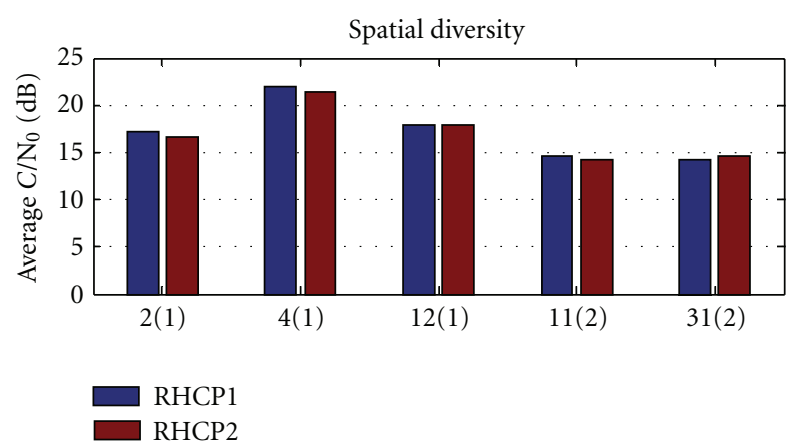

(b)

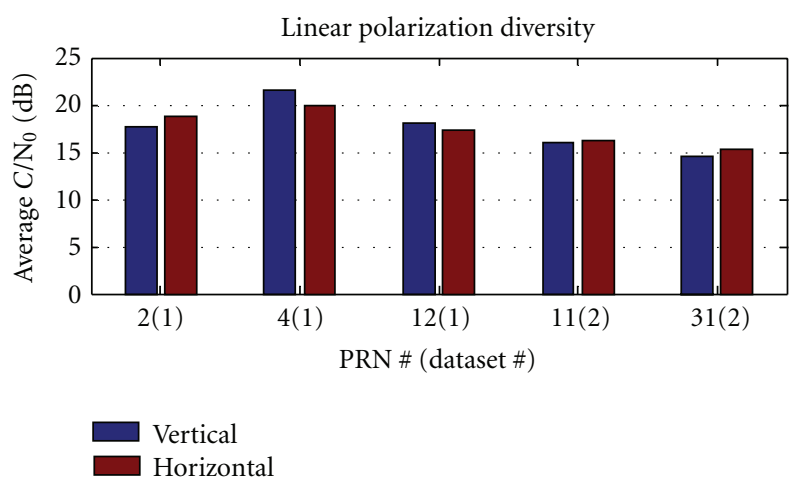

(c)

FIGURE 9: Average input SNR for different diversity structures. 


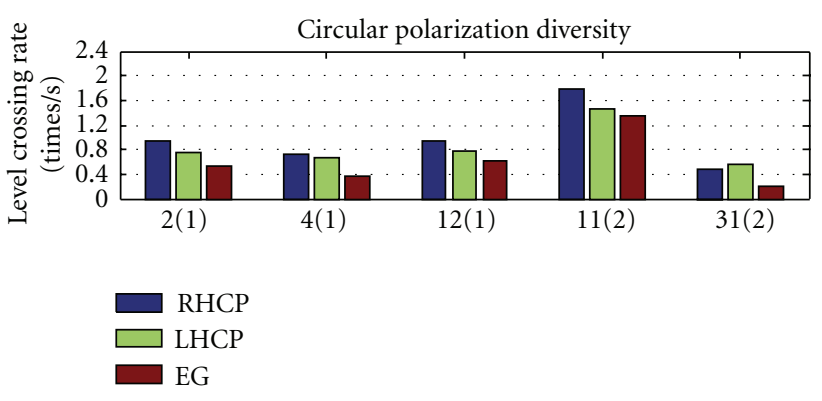

(a)

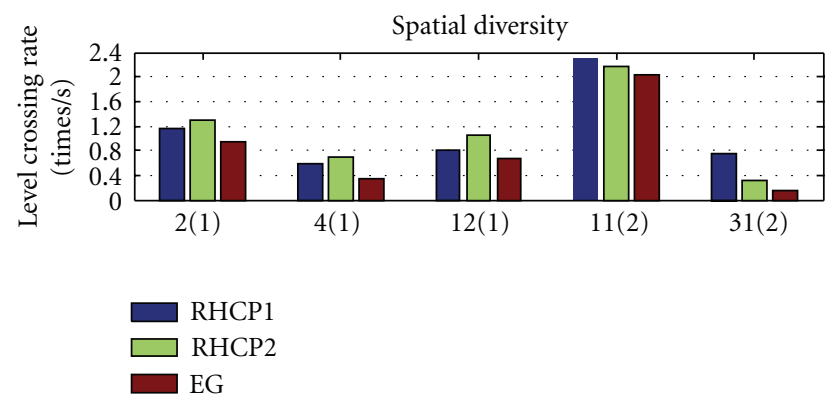

(b)
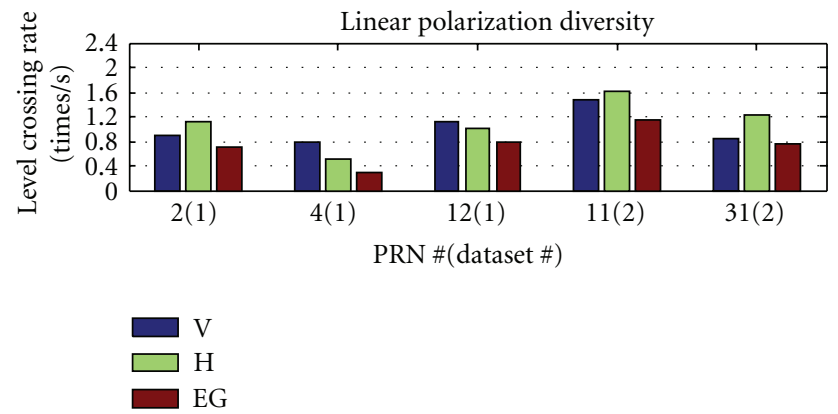

(c)

FIGURe 10: LCR analysis for single branches and different diversity structures $(\mathrm{V}$ and $\mathrm{H}$ stand for vertical and horizontal linearly polarized antennas).

The threshold for the LCR and AFD quantification procedure can be defined based on the level (signal envelope) or signal SNR. Since in GPS applications the SNR values has been extensively used, in this work the signal post-SNR is utilized for the LCR and AFD quantification. In statistical detection theory, the post-SNR can be quantified by the deflection coefficient as [24]

$$
d^{2}=\frac{\left[E\left(T(x) \mid H_{1}\right)-E\left(T(x) \mid H_{0}\right)\right]^{2}}{\operatorname{var}\left(T(x) \mid H_{0}\right)},
$$

where $E(\cdot)$ is the expectation operator, $E\left(T(x) \mid H_{1}\right)$ is the peak of the correlation function, $E\left(T(x) \mid H_{0}\right)$ is the mean value of the test statistics under $H_{0}$, and $\operatorname{var}\left(T(x) \mid H_{0}\right)$ gives the variance of the test statistics under $H_{0}$. The AFD and LCR are quantified for all PRNs and datasets for a specific threshold, which is $10 \mathrm{~dB}$ less than the signal root mean square (rms). Figure 10 shows the LCR values for the utilized diversity schemes using the EG combiner. The combined

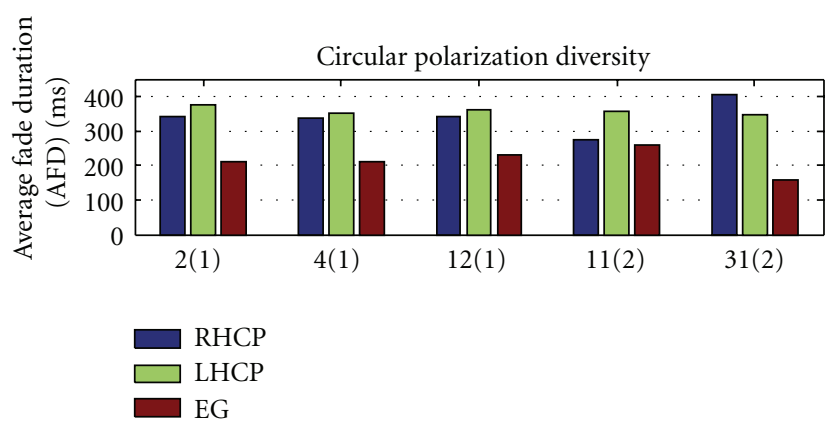

(a)

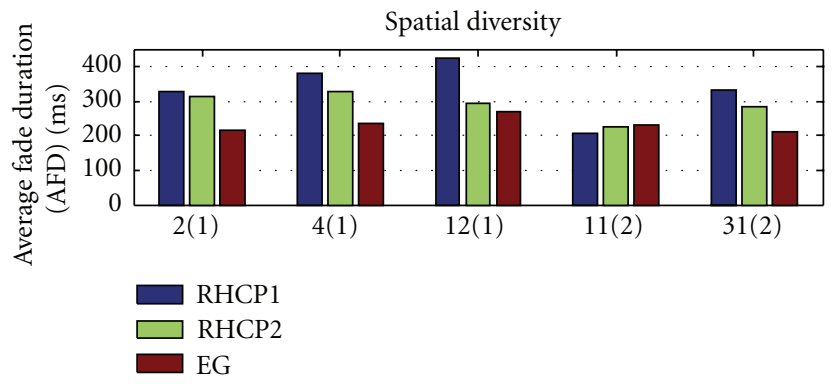

(b)
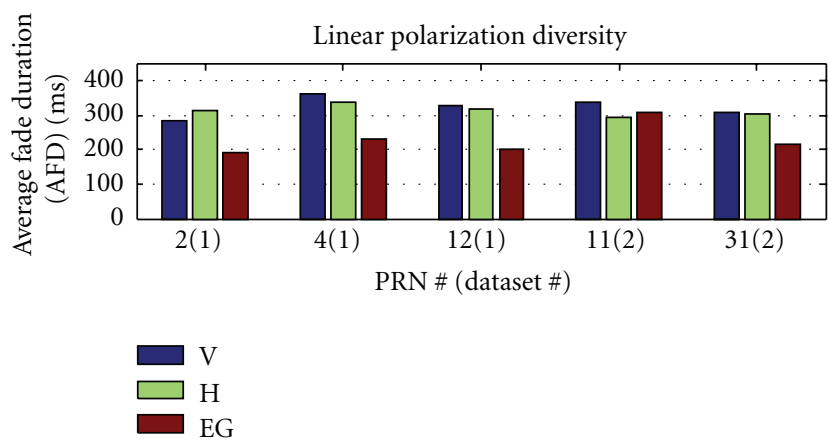

(c)

FIGURE 11: AFD analysis for single branches and different diversity structures ( $\mathrm{V}$ and $\mathrm{H}$ stand for vertical and horizontal linearly polarized antennas).

signals in all diversity structures show less fading compared to the single antenna cases. Figure 11 shows the measured AFD values and the significant improvement in the combined signals in all diversity structures. According to these results, overall all diversity structures result in the same improvement in the fading mitigation.

4.2.4. Diversity Gain. The diversity gain for a specific design point in terms of $P_{\mathrm{FA}}$ and $P_{D}$ is quantified as the reduction in the input average SNR of the diversity branches to provide the same detection performance utilizing a single channel receiver. The measured diversity gains for different diversity systems are shown in Figure 12 where the diversity gain is measured for $P_{\mathrm{FA}}=0.01$. The average diversity gain of the collected data for circular polarization, spatial, and linear polarization diversity structures is $3.7 \mathrm{~dB}, 2.8 \mathrm{~dB}$, and $2.2 \mathrm{~dB}$, respectively. As discussed previously, since in indoor environments the GNSS signal is reshaped to the elliptically 


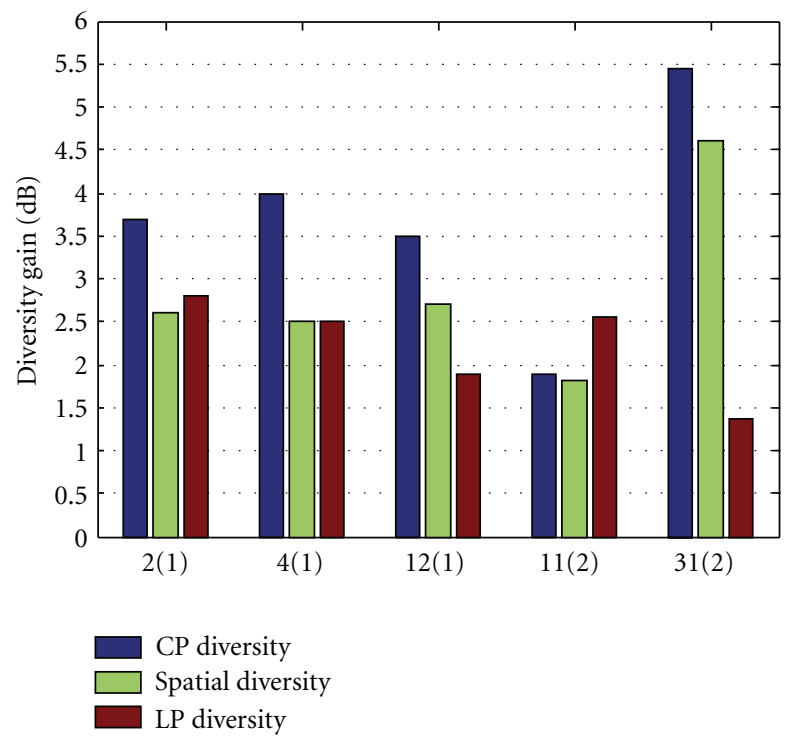

FIgURE 12: Diversity gain for various diversity schemes.

polarized waves which can be observed by the RHCP and LHCP antennas, the polarization diversity employing the RHCP and LHCP antennas improves the GPS signal detectability and leads to higher performance. However, spatial diversity systems take only the power of the RHCP component of the received signals, which leads to lower processing gain.

\section{Conclusions}

Spatial and polarization diversity systems were employed to combat fading in dense multipath environments. Performance enhancements arising from utilizing the spatial and polarization diversity techniques were first studied theoretically. It was shown that the detection performance of the EG combiner is similar to that of the EC for low correlation coefficients values (less than 0.4) and low power differences between diversity branches. Experiments were performed to compare polarization and spatial diversity structures. It was observed that both spatial and polarization diversity result in sufficiently low correlation coefficients between the received signals with a low power difference between input branches. However, the received signal power through the LHCP antenna outweighs the signal power received by the RHCP antenna. According to the results for the target detection design point in term of $P_{\mathrm{FA}}$ and $P_{D}$, the polarization diversity leads to higher diversity gain in indoor environments compared to the spatial diversity schemes. The diversity gain can reach up to $5 \mathrm{~dB}$ for the practical range of detection design point. LCR and AFD values, representing the secondorder statistics of the fading channel, were also measured for different diversity branches.

\section{References}

[1] S. Aguire, L. H. Loew, and and Y. Lo, "Radio propagation into buildings at 912,1920, and $5990 \mathrm{MHz}$ using microcells," in Proceedings of the 3rd International Conference on Universal Personal Communications (ICUPC '92), pp. 129-134, 1994.

[2] J. Horikoshi, K. Tanaka, and T. Morinaga, "1.2 GHz Band wave propagation measurements in concrete Buildings for indoor Radio communications," IEEE Transactions on Vehicular Technology, vol. 35, no. 4, pp. 146-152, 1986.

[3] M. E. Cannon, G. Lachapelle, G. MacGougan, P. Boulton, and A. Read, Mobile Telephony: Testing a High Sensitivity GPS Receiver in Weak Signal Environments, GPS World, 2003.

[4] G. Lachapelle, H. Kuusniemi, D. T. H. Dao, G. MacGougan, and M. E. Cannon, "HSGPS signal analysis and performance under various indoor conditions," in Proceedings of the 16th International Technical Meeting of the Satellite Division of The Institute of Navigation (ION GPS/GNSS '03), pp. 1171-1184, Portland, Ore, USA, September 2003.

[5] G. D. MacGougan, High sensitivity GPS performance analysis in degraded signal environments, M.S. thesis, Department of Geomatics Engineering, University of Calgary, Canada, 2003.

[6] S. Shanmugam, New enhanced sensitivity detection techniques for GPS L1 C/A and modernized signal acquisition, Ph.D. thesis, Department of Geomatics Engineering, The University of Calgary, 2008.

[7] J. B. Bancroft, G. Lachapelle, M. E. Cannon, and M. G. Petovello, "Twin IMU-HSGPS integration for pedestrian navigation," in Proceedings of the 21st International Technical Meeting of the Satellite Division of The Institute of Navigation (ION GNSS '08), Savannah, Ga, USA, September 2008.

[8] G. Gao and G. Lachapelle, "A novel architecture for ultratight HSGPS-INS integration," Journal of Global Positioning Systems, vol. 7, no. 1, pp. 46-61, 2008.

[9] A. Singer, "A comparison of space diversity and polarization diversity receive systems for cellular and PCS systems," 1998, http://www.rfsworld.com/index.php?indexVal=2\&p=183.

[10] M. Zaheri, A. Broumandan, C. O’Driscoll, and G. Lachapelle, "Enhanced GNSS indoor signal detectability using polarization diversity," in Proceedings of the 22nd International Technical Meeting of The Satellite Division of the Institute of Navigation (ION GNSS '09), Savannah, Ga, USA, September 2009.

[11] J. Nielsen, S. K. Shanmugam, M. U. Mahfuz, and G. Lachapelle, "Enhanced detection of weak GNSS signals using spatial combining," Navigation, Journal of the Institute of Navigation, vol. 56, no. 2, pp. 83-95, 2009.

[12] J. S. Colburn, Y. Rahmat-Samii, M. A. Jensen, and G. J. Pottie, "Evaluation of personal communications dual-antenna handset diversity performance," IEEE Transactions on Vehicular Technology, vol. 47, pp. 737-744, 1998.

[13] H. Bolcskei, D. Gesbert, C. B. Papadias, and A.-J. van der Veen, Space-Time Wireless Systems-From Array Processing to MIMO Communications, Cambridge University Press, Cambridge, UK, 2006.

[14] S. Hyeon, Y. Yun, H. Kim, and S. Choi, "Phase diversity for an antenna-array system with a short interelement separation," IEEE Transactions on Vehicular Technology, vol. 57, no. 1, pp. 206-214, 2008.

[15] R. M. Narayanan, K. Atanassov, V. Stoiljkovic, and G. R. Kadambi, "Polarization diversity measurements and analysis for antenna configurations at $1800 \mathrm{MHz}$," IEEE Transactions on Antennas and Propagation, vol. 52, no. 7, pp. 1795-1810, 2004.

[16] A. M. D. Turkmani, A. A. Arowojolu, P. A. Jefford, and C. J. Kellett, "Experimental evaluation of the performance of two-branch space and polarization diversity schemes at 
1800 MHz," IEEE Transactions on Vehicular Technology, vol. 44, no. 2, pp. 318-326, 1995.

[17] J. F. Lemieux, M. S. El-Tanany, and H. M. Hafez, "Experimental evaluation of space/frequency/polarization diversity in the indoor wireless channel," IEEE Transactions on Vehicular Technology, vol. 40, no. 3, pp. 569-574, 1991.

[18] A. Broumandan, Enhanced narrowband signal detection and estimation with a synthetic antenna array for location applications, Ph.D. thesis, Department of Geomatics Engineering, The University of Calgary, Canada, 2009.

[19] E. D. Kaplan and C. Hegarty, Understanding GPS Principles and Applications, Artech House, 2nd edition, 2006.

[20] T. S. Rappaport, Wireless Communications: Principles and Practice, Prentice Hall, Upper Saddle River, NJ, USA, 2nd edition, 2002.

[21] C. Yang and A. Porter, "GPS multipath estimation and mitigation via polarization sensing diversity: parallel iterative cross cancellation," in Proceedings of the 18th International Technical Meeting of the Satellite Division of The Institute of Navigation (ION GNSS '05), pp. 2707-2719, San Diego, Calif, USA, September 2005.

[22] J. Polívka, "Measuring the permittivity of concrete at millimeter waves," International Journal of Infrared and Millimeter Waves, vol. 17, no. 10, pp. 1673-1683, 1996.

[23] C. Balanis, Antenna Theory, Analysis and Design, John Wiley \& Sons, New York, NY, USA, 1997.

[24] S. M. Kay, Fundamentals of Statistical Signal Processing"Part 2: Detection Theory, Prentice Hall, Upper Saddle River, NJ, USA, 1998.

[25] A. Broumandan, J. Nielsen, and G. Lachapelle, "Enhanced detection performance of indoor GNSS signals based on synthetic aperture," IEEE Transactions on Vehicular Technology, vol. 59, no. 6, Article ID 5454362, pp. 2711-2724, 2010.

[26] M. Zaheri, Enhanced GNSS signal detection performance utilizing polarization diversity, M.S. thesis, Department of Geomatics Engineering, The University of Calgary, Canada, 2011.

[27] J. D. Parsons, The Mobile Radio Propagation Channel, John Wiley \& Sons, New York, NY, USA, 2nd edition, 2000.

[28] "ANTCOM Vertical/Horizontal or RHCP/LHCP L1/L2 GPS Antennas," 2008, http://www.antcom.com/documents/catalogs/RHCP-LHCP-V-H-L1L2GPSAntennas.pdf.

[29] C. O’Driscoll, D. Borio, M. G. Petovello, T. Williams, and G. Lachapelle, "The soft approach: a recipe for a multi-system, multi-frequency GNSS receiver," Inside GNSS Magazine, vol. 4, no. 5, pp. 46-51, 2009.

[30] N. Blaunstein and J. B. Andersen, Multipath Phenomena in Cellular Networks, Artech House, 2002. 

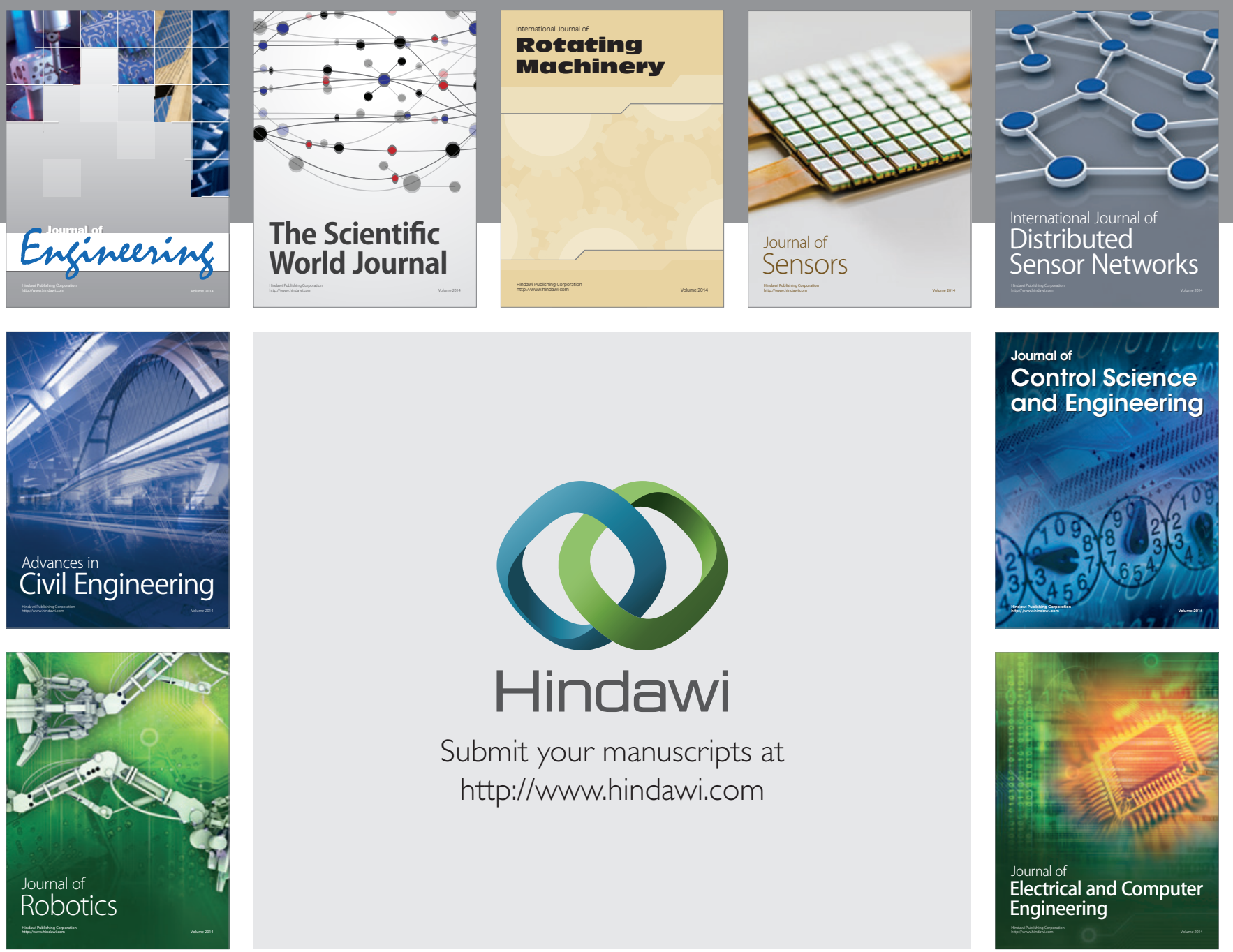

Submit your manuscripts at

http://www.hindawi.com
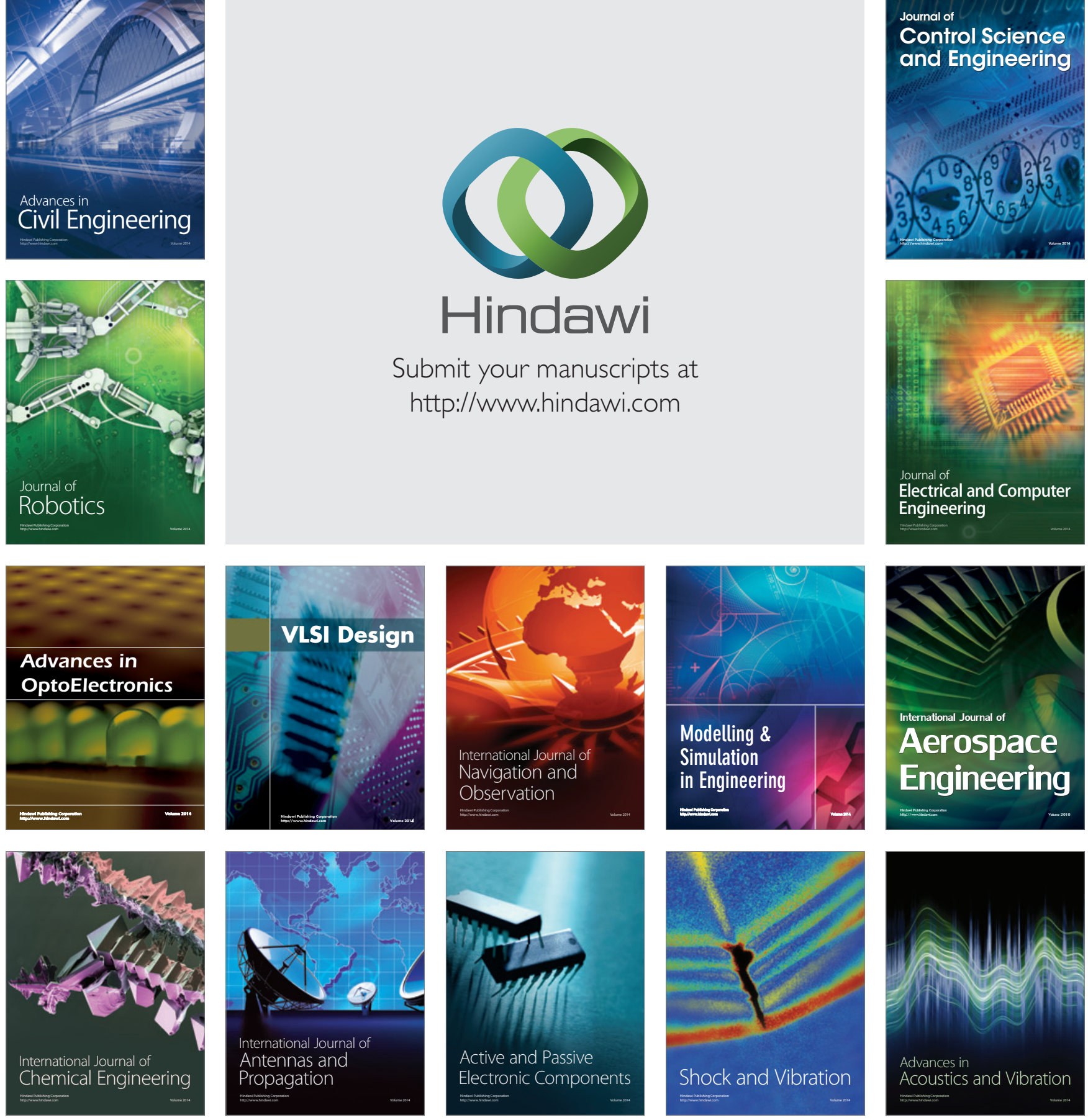